\title{
Prie et travaille
}

La vieille règle des Bénédictins «ora et labora» était la voie à suivre pour une vie partagée entre contemplation et ouvrage: une harmonie monacale. Même la société de partage du travail éprouve des difficultés à trouver l'équilibre entre les exigences les plus diverses. On parle beaucoup de «work-life balance» (équilibre vie-travail), une mauvaise notion pour un vrai problème. Il vaudrait mieux parler $\mathrm{d}^{\prime}$ «équilibre du temps», du temps à répartir judicieusement entre la prestation et le travail d'une part, les amis et la famille, ainsi que le corps et l'interprétation des choses d'autre part [1]. L'éditorial du Forum médical suisse [2] critique à bon droit le fait d'exclure le travail d'une vie considérée comme réussie, comme si le travail n'était pas une valeur en soi.

Il se cache toutefois bien plus dans cette formule qu'une attitude négative contre le travail, la peur d'une charge trop intensive de travail ou la volonté de prendre une retraite anticipée. Les médecins sont-ils particulièrement bien placés, en tant que groupe professionnel, pour indiquer à leurs patients la juste mesure? N'est-ce pas plutôt que dans les sociétés modernes de services, le travail et les loisirs ont fortement changé d'une génération à l'autre? L'image de la carrière n'a plus longue vie, les titres universitaires ont perdu de leur prestige, les carrières sont acculées à l'insécurité et les valeurs solides du prestige sont dépassées. La pression interne de la concurrence a augmenté en proportion de la vitesse prise par les technologies et la globalisation. De nouveaux rôles types tendent à remplacer les anciens. Si l'on veut accueillir dans le monde du travail plus de femmes et de personnes âgées ou augmenter les taux de naissance, il faut changer les structures familiales et professionnelles d'antan. Il est impossible de revenir à des modèles patriarcaux de la période préindustrielle. De plus, les sociétés développées n'ont bientôt plus de travail, car un nombre de biens et de prestations de plus en plus grand est produit par un nombre toujours plus petit d'artisans dans des temps toujours plus courts.

L'économie actuelle n'a pas encore de réponse

1 Seiwert LJ. Wenn Du es eilig hast, gehe langsam. Frankfurt am Main/ New York: Campus; 1998.

2 Krapf R. Das Fehlkonzept der «Work-Life-Balance». Schweiz Med Forum. 2007;7(23):495. d'un côté et explosant de l'autre - ceux des chefs d'entreprise - contribuent à dévaloriser encore le revenu des travailleurs normaux. Lorsque l'équilibre n'existe plus, alors entrent en jeu l'augmen- tation des frais de maladie, les rentes précoces, les pertes de production et les fluctuations de personnel. De nombreuses entreprises misent donc sur des formes d'organisation souples et sur le management individuel. Elles offrent des mesures visant une qualité de vie meilleure grâce à l'aménagement du temps de travail, aux congés sabbatiques, à l'assistance des enfants dans l'entreprise, aux congés parentaux, aux possibilités de formation complémentaire ou d'entraînement, au télétravail et aux programmes de mise en forme et bien-être. A cet égard, les institutions de la santé publique sont à la traîne, même si leurs déficits sont les plus visibles. Nous savons tous que le personnel soignant et les médecins sont épuisés et à la limite du burn-out. Il est aussi largement prouvé que la gent masculine est particulièrement sujette à la dépendance au travail, dans une aspiration maladive de reconnaissance, malgré tous les côtés négatifs que cela comporte.

Le temps des loisirs n'est plus le même non plus. On programme déjà les enfants avant l'âge scolaire et l'offre en surnombre des objets de consommation provoque la fébrilité et le stress. «Davantage de temps pour vivre» promet la Migros à ses consommateurs de plats préparés et déjà, un laps de temps libre nous est volé. A la fin, même les activités joyeuses deviennent des fardeaux. L'équilibre entre vie professionnelle et vie privée devient de plus en plus difficile à trouver.

Une «génération de stage» qui vit dans des conditions de travail non régulières a les mêmes soucis que les jeunes médecins entre pression de la rationalisation et arrêt de la pratique libre. Les chants de sirène vantant les hiérarchies plates et l'autoresponsabilité accrue n'ont que peu en commun avec l'autonomie vue comme une tâche à vie. L'une de ces approches prône le développement intérieur d'une existence indépendante et volontaire, l'autre plus d'efficacité et de contrôle de soi dans l'intérêt de la production. Ces vertus sont essentielles à qui exige de ses collaborateurs la motivation et la loyauté. «Prie et travaille», c'est aujourd'hui le «work-life balance» sous une forme devenue profane, difficile à atteindre, mais ô combien actuelle, comme à l'époque de Saint Benoît de Nursie.

Erhard Taverna 